\title{
THE CONTINUITY OF DERIVATIONS AND MODULE HOMOMORPHISMS
}

\author{
G. A. WILLIS \\ (Received 14 February 1983; revised 25 June 1984) \\ Communicated by G. Brown
}

\begin{abstract}
This paper is concerned with the problem of automatic continuity of derivations from group algebras $L^{1}(G)$, where $G$ is a locally compact group, and convolution algebras $L^{1}(\omega)$, where $\omega$ is a weight function. In the case of group algebras, it is shown that either the problem reduces to the case when $G$ is the free group on a countably infinite number of generators or there is a non-discrete group $G$ with a discontinuous $l^{1}(G)$-bimodule homomorphism from $L^{1}(G)$. It is also shown that every derivation from $L^{1}(G)$ to a commutative $L^{1}(G)$-bimodule is continuous. Similar results are obtained for weighted convolution algebras.
\end{abstract}

1980 Mathematics subject classification (Amer. Math. Soc.): 46 H 25.

\section{Introduction}

It is not known whether derivations from group algebras $L^{1}(G)$ or weighted convolution algebras $L^{1}(\omega)$ to Banach bimodules over the respective algebras are automatically continuous. For a discussion of the problem as to whether they are, see Section 10 in [3] and, in particular, questions 22 and 24. The present paper is concerned with developing some techniques which may be used to tackle this problem and with applying them to some particular cases.

A related question is raised in Section 2, namely whether every left $l^{1}(G)$-module (or $l^{1}(\omega)$-module) homomorphism from $L^{1}(G)$ (respectively $L^{1}(\omega)$ ) to an arbitrary left Banach $l^{1}(G)$-module $\left(l^{1}(\omega)\right.$-module) is continuous. Some evidence is presented which suggests that all such homomorphisms are continuous. How

(c) 1986 Australian Mathematical Society $0263-6115 / 86 \$ A 2.00+0.00$ 
this is related to the automatic continuity problem for derivations from $L^{1}(G)$ is shown in Section 4 (Theorem 4.1). In particular, it is shown that either the homomorphisms problem has a negative solution or the derivations problem reduces to the case when $G$ is the free group on a countably infinite number of generators. In other words, if the homomorphisms problem has a positive solution, then the topology on $G$ is not relevant to the derivations problem. An analogous result is proved for $L^{1}(\omega)$.

The other main result of Section 4 (Theorem 4.3) is a solution of a special case of the derivations problem for group algebras. It is shown that every derivation from $L^{1}(G)$ to a commutative Banach $L^{1}(G)$-bimodule is continuous. Some lemmas required in Section 4 are proved in Section 3. These lemmas are based on parts of my Ph D thesis, which was completed at the University of Newcastle upon Tyne under the supervision of Professor B. E. Johnson. I would like to thank Professor Johnson for his supervision and many helpful suggestions.

\section{Weight functions and other definitions}

We begin by describing the convolution algebras on groups and on the positive half-line with which we shall be working.

Throughout, $G$ will denote a locally compact group. All notation concerning group algebras will be as in [9] except that we shall identify certain algebras which are shown to be isomorphic there. For example, $C_{0}^{*}(G)$ will be identified with $M(G)(19.12), l^{1}(G)$ with $M_{a}(G)(19.18)$ and $l^{1}(G)$ with $M_{d}(G)(19.15)$. For each $x$ in $G$ we shall denote the point mass at $x$ by $\bar{x}$.

Two notions of weight function have been used by varioous authors in the definition of weighted convolution algebras. In both cases a weight function is defined to be a function $\omega:[0, \infty) \rightarrow \mathbf{R}^{+}$such that

$$
\omega(s+t) \leqslant \omega(s) \omega(t), \quad(s, t \in[0, \infty)) .
$$

A function satisfying (1.1) is said to be submultiplicative. However, in [7], and [3] for example, it is required that $\omega$ should also be continuous and that $\omega(0)=1$, whereas in [1] the only requirement is that $\omega$ should be measurable.

In any case, a weight function $\omega$ is said to be bounded if there is an $M>0$ such that $\omega(t) \leqslant M,(t \in[0, \infty))$ and to be bounded near zero if there is an $M>0$ and $\delta>0$ such that

$$
\omega(t) \leqslant M \quad(t \in[0, \delta]) .
$$

If $\omega$ is continuous, then it is automatically bounded near zero.

By Theorem 7.6 .5 of [10], $\lim _{t \rightarrow \infty} \omega(t)^{1 / t}$ always exists. If this limit is equal to zero, then $\omega$ is said to be a radical weight function. 
Two weight functions, $\omega_{1}$ and $\omega_{2}$, are said to be equivalent if there are constants $m, M>0$ with

$$
m \omega_{1}(t) \leqslant \omega_{2}(t) \leqslant M \omega_{1}(t) \quad(t \in[0, \infty)) .
$$

For either notion of weight function, define the weighted convolution algebra $L^{1}(\omega)$ to be the set of equivalence classes of complex-valued measurable functions $f$ on $[0, \infty)$ such that

$$
\|f\|=\int_{0}^{\infty}|f(t)| \omega(t) d t<\infty
$$

where two functions are equivalent if they are equal almost everywhere. Define $M(\omega)$ to be the set of complex, regular, Borel measures $\mu$ on $[0, \infty)$ such that

$$
\|\mu\|=\int_{0}^{\infty} \omega(t) d|\mu|(t)<\infty .
$$

Then $L^{1}(\omega)$ and $M(\omega)$ are Banach algebras with convolution as their product (see [1] and [7]). We will identify $L^{1}(\omega)$ with the closed, two-sided ideal in $M(\omega)$ consisting of measures which are absolutely continuous with respect to Lebesgue measure. Under this identification, Theorem 1.4 in [7] shows that, if $\omega$ is a continous weight, then the multiplier algebra of $L^{1}(\omega)$ is isomorphic to $M(\omega)$.

Certain properties of the weight function correspond to properties of the weighted convolution algebra. For example, $\omega$ is a radical weight if and only if $L^{1}(\omega)$ is a radical Banach algebra and, if $\omega$ is bounded near zero, then $L^{1}(\omega)$ has a bounded approximate identity (see [1]). By comparing the norms of point masses, we see that two weights $\omega_{1}$ and $\omega_{2}$ are equivalent if and only if $M\left(\omega_{1}\right)=M\left(\omega_{2}\right)$. Further, if $\omega_{1}$ and $\omega_{2}$ are equivalent, then it is clear that $L^{1}\left(\omega_{1}\right)=L^{1}\left(\omega_{2}\right)$.

It is at this point that it begins to matter whether we take weights to be continuous or merely measurable. If $\omega_{1}$ and $\omega_{2}$ are continuous and $L^{1}\left(\omega_{1}\right)=$ $L^{1}\left(\omega_{2}\right)$, then it may be shown that $\omega_{1}$ and $\omega_{2}$ are equivalent. However, there are inequivalent measurable $\omega_{1}$ and $\omega_{2}$ with $L^{1}\left(\omega_{1}\right)=L^{1}\left(\omega_{2}\right)$.

For such a pair of weights $L^{1}\left(\omega_{1}\right)=L^{1}\left(\omega_{2}\right)$ but $M\left(\omega_{1}\right) \neq M\left(\omega_{2}\right)$. Hence, as it stands, Theorem 1.4 in [7] does not generalize from continuous to measurable weights because there is no natural candidate for the multiplier algebra. On the other hand, there ought to be some sort of generalization of this theorem to weights which are bounded near zero because the most important feature of its proof is the existence of a bounded approximate identity in $L^{1}(\omega)$.

This difficulty can be met by making an appropriate choice for the definition of weight function. This choice is suggested by Proposition 1.1 below, the first part of which is due to S. Grabiner [8, Corollary 3.6]. For this, define the Sorgenfrey topology on $[0, \infty)$ to be that which has the base $\{[x, y) \mid x, y \in[0, \infty)\}$ (see [5], ex. 
1.2.2). It is clear that a complex-valued function $f$ on $[0, \infty)$ is Sorgenfrey continuous if and only if $\lim _{h \rightarrow 0^{+}} f(t+h)=f(t),(t \in[0, \infty))$. Every Sorgenfrey open set is a Borel set. Hence a Sorgenfrey continuous function is Borel.

1.1. Proposition. Let $\omega$ be a measurable submultiplicative function on $[0, \infty)$ which is bounded near zero. Then there is a Sorgenfrey continuous submultiplicative function $\bar{\omega}$ on $[0, \infty)$ such that $L^{1}(\omega)=L^{1}(\bar{\omega})$.

If $\omega^{\prime}$ is any Sorgenfrey continuous submultiplicative function with $L^{1}\left(\omega^{\prime}\right)=L^{1}(\omega)$, then $\omega^{\prime}$ is equivalent to $\bar{\omega}$.

Proof. The first part is Corollary 3.6 of [8]. To prove the second part, let $\omega^{\prime}$ be a Sorgenfrey continuous submultiplicative function such that $L^{1}\left(\omega^{\prime}\right)=L^{1}(\omega)$. Define, for $f$ in $L^{1}(\omega)$,

$$
\|f\|=\int_{0}^{\infty}|f(t)| \bar{\omega}(t) d t
$$

and

$$
\|f\|\left|=\int_{0}^{\infty}\right| f(t) \mid \omega^{\prime}(t) d t .
$$

Then $\|\cdot\|$ and $\|\cdot\|$ are Banach algebra norms on $L^{1}(\omega)$ and are thus equivalent by the argument given in [3, Theorem 7.6]. Hence there are constants $m, M$ such that

$$
m\|f\| \leqslant\|f\| \leqslant M\|f\| \quad\left(f \in L^{1}(\omega)\right) .
$$

Let $t$ be in $[0, \infty)$ and for each $h>0$ define

$$
f_{h}(s)= \begin{cases}h^{-1}, & t \leqslant s \leqslant t+h, \\ 0, & \text { otherwise. }\end{cases}
$$

Then, because $\bar{\omega}$ and $\omega^{\prime}$ are Sorgenfrey continuous,

$$
\begin{aligned}
\lim _{h \rightarrow 0^{+}}\left\|f_{h}\right\| & =\lim _{h \rightarrow 0^{+}} \int_{0}^{\infty}\left|f_{h}(s)\right| \bar{\omega}(S) d s \\
& =\lim _{h \rightarrow 0^{+}} \int_{t}^{t+h} h^{-1} \bar{\omega}(s) d s \\
& =\bar{\omega}(t),
\end{aligned}
$$

and $\lim _{h \rightarrow 0^{+}}\left\|\mid f_{h}\right\| \|=\omega^{\prime}(t)$. Therefore, by $(1.6), m \omega^{\prime}(t) \leqslant \bar{\omega}(t) \leqslant M \omega^{\prime}(t),(t \in$ $[0, \infty))$, and so $\omega^{\prime}$ and $\bar{\omega}$ are equivalent.

In view of Theorem 1.1 we now define a weight function to be a Sorgenfrey continuous submultiplicative function $\omega:[0, \infty) \rightarrow \mathbf{R}^{+}$. Note that such weight functions $\omega$ are always bounded near zero and so the weighted convolution algebra $L^{1}(\omega)$ will always have a bounded approximate identity. 
With this notion of weight function we have that $\omega_{1}$ and $\omega_{2}$ are equivalent if and only if $L^{1}\left(\omega_{1}\right)=L^{1}\left(\omega_{2}\right)$. Also, we recover all the weighted convolution algebras which arise from measurable submultiplicative functions which are bounded near zero. However, this definition of weight function is still more restrictive than that used in [1] because in that paper weights are allowed to be unbounded near zero.

A version of Theorem 1.4 in [7] may now be proved for this more general notion of weight function. By a multiplier on a Banach algebra $A$ is meant a continuous linear operator $T: A \rightarrow A$ such that $T(a b)=T(a) b,(a, b \in A)$. The set of all multipliers on $A$ will be denoted by $\mathscr{M}(A)$. It is a closed subalgebra of the algebra of all bounded linear operators on $A$.

For each $\mu$ in $M(\omega)$, let $\mathscr{T}_{\mu}$ be the operator on $L^{1}(\omega)$ defined by

$$
\mathscr{T}_{\mu}(f)=\mu * f \quad\left(f \in L^{1}(\omega)\right) .
$$

Then it is clear that $\mathscr{T}$ is a continuous homomorphism from $M(\omega)$ into $\mathscr{M}\left(L^{1}(\omega)\right)$. We will show that $\mathscr{T}$ is a bijection. As in [7], the proof will be modelled on Wendel's proof that $M(G)$ is the multiplier algebra of $L^{1}(G)$ (see [9, Theorem 35.5]). For this, it is necessary to represent $M(\omega)$ as a dual space.

Let $C_{0}(\omega)$ be the space of all continuous functions $\psi$ on $[0, \infty)$ with

$$
\|\psi\|=\sup _{t \in[0, \infty)}\left|\psi(t) \omega(t)^{-1}\right|<\infty
$$

and

$$
\lim _{t \rightarrow \infty}\left|\psi(t) \omega(t)^{-1}\right|=0
$$

Then $\left(C_{0}(\omega),\|\cdot\|\right)$ is a Banach space.

1.2 Lemma. Let $\omega$ be a weight function. Then $C_{0}(\omega)^{*}$ (the dual space of $C_{0}(\omega)$ ) is isomorphic to $M(\omega)$.

Proof. Define, for each $\mu$ in $M(\omega)$,

$$
m_{\mu}(\psi)=\int_{0}^{\infty} \psi d \mu \quad\left(\psi \in C_{0}(\omega)\right)
$$

Then,

$$
\begin{aligned}
\left|m_{\mu}(\psi)\right| & \leqslant \int_{0}^{\infty}|\psi| d|\mu|=\int_{0}^{\infty}\left(|\psi| \omega^{-1}\right) \omega d|\mu| \\
& \leqslant\left(\sup _{[0, \infty)}|\psi| \omega^{-1}\right) \int_{0}^{\infty} \omega d|\mu|=\|\psi\|\|\mu\| .
\end{aligned}
$$

Hence, $m_{\mu}$ is in $C_{0}(\omega)^{*}$ and $\left\|m_{\mu}\right\| \leqslant\|\mu\|$. 
Both $\omega$ and $\omega^{-1}$ are bounded on $[0, t]$ for every $t$ in $[0, \infty)$ and so the subspace $C_{t}$ of $C_{0}(\omega)$ consisting of functions with support in $[0, t)$ is isomorphic to $C_{0}[0, t)$. Hence, if $m$ is in $C_{0}(\omega)^{*}$, then, by the Riesz Representation Theorem [15, Theorem 6.19] there is a regular Borel measure $\mu$ on $[0, \infty)$ such that $m(\psi)=$ $\int_{0}^{t} \psi(s) d \mu(s)=\int_{0}^{\infty} \psi d \mu,\left(\psi \in C_{t}\right)$. It is clear that $\mu$ may be chosen independently of $t$. We will show that $\mu$ is in $M(\omega)$ and $m=m_{\mu}$.

For each $\varepsilon$ in $(0,1)$ define a function $\omega_{\varepsilon}$ on $[0, \infty)$ by

$$
\omega_{\varepsilon}(t)=\frac{1}{\varepsilon} \int_{0}^{\varepsilon} \omega(t+s) d s \quad(t \in[0, \infty)) .
$$

Then $\omega_{\varepsilon}$ is continuous on $[0, \infty)$. Since $\omega$ is submultiplicative we have $\omega_{\varepsilon} \leqslant M \omega$, where $M$ is an upper bound for $\omega$ on $[0,1]$ and, since $\omega$ is Sorgenfrey continuous, $\omega_{\varepsilon}$ converges pointwise to $\omega$ as $\varepsilon \rightarrow 0$.

Now consider

$$
\int_{[0, t)} \omega d|\mu|=\sup \left\{\left|\int_{0}^{t} \psi \omega d \mu\right| \mid \psi \in C_{0}[0, t),\|\psi\|_{\infty} \leqslant 1\right\} .
$$

We have, for each $\psi$ in $C_{0}[0, t)$, that $\psi \omega$ is in $L^{1}(|\mu|),\left|\psi \omega_{\varepsilon}\right| \leqslant|M \psi \omega|$, and $\psi \omega$ converges pointwise to $\psi \omega$. Hence, by the dominated convergence theorem,

$$
\int_{0}^{t} \psi \omega d \mu=\lim _{\varepsilon \rightarrow 0} \int_{0}^{t} \psi \omega_{\varepsilon} d \mu
$$

Thus

$$
\begin{aligned}
\int_{[0, t)} \omega d|\mu| & =\sup \left\{\left|\lim _{\varepsilon \rightarrow 0} \int_{0}^{t} \psi \omega_{\varepsilon} d \mu\right| \mid \psi \in C_{0}[0, t),\|\psi\|_{\infty} \leqslant 1\right\} \\
& \leqslant \varlimsup_{\varepsilon \rightarrow 0}\left(\sup \left\{\left|\int_{0}^{t} \psi \omega_{\varepsilon} d \mu\right| \mid \psi \in C_{0}[0, t),\|\psi\|_{\infty} \leqslant 1\right\}\right) \\
& =\varlimsup_{\varepsilon \rightarrow 0}\left(\sup \left\{\left|\int_{0}^{t} \psi d \mu\right|\left\|\psi \in C_{0}[0, t),\right\| \psi \omega_{\varepsilon}^{-1} \|_{\infty} \leqslant 1\right\}\right) .
\end{aligned}
$$

The last equality holds because $\omega_{\varepsilon}^{-1}$ is continuous.

Since $\omega_{\varepsilon} \leqslant M \omega$, we have that, if $\left\|\psi \omega_{\varepsilon}^{-1}\right\|_{\infty} \leqslant 1$, then $\left\|\psi \omega^{-1}\right\| \leqslant M$. Hence

$$
\begin{aligned}
\sup \left\{\mid \int_{0}^{t} \psi d \mu \| \psi\right. & \left.\in C_{0}[0, t),\left\|\psi \omega_{\varepsilon}^{-1}\right\|_{\infty} \leqslant 1\right\} \\
& \leqslant \sup \left\{\left|\int_{0}^{t} \psi d \mu\right|\left\|\in C_{0}[0, t),\right\| \psi \omega^{-1} \|_{\infty} \leqslant M\right\} \\
& =M\left(\sup \left\{\mid \int_{0}^{t} \psi d \mu\left\|\psi \in C_{0}[0, t),\right\| \psi \omega^{-1} \|_{\infty} \leqslant 1\right\}\right) .
\end{aligned}
$$


It follows that

$$
\begin{aligned}
\int_{[0, t)} \omega d|\mu| & \leqslant M\left(\sup \left\{\mid \int_{0}^{t} \psi d \mu\left\|\psi \in C_{0}[0, t),\right\| \psi \omega^{-1} \| \leqslant 1\right\}\right) \\
& \leqslant M\|m\|,
\end{aligned}
$$

by definition of the norm on $C_{0}(\omega)^{*}$. Therefore, $\mu$ is in $M(\omega)$ and

$$
\|\mu\|=\int_{0}^{\infty} \omega d|\mu|=\lim _{t \rightarrow \infty} \int_{[0, t)} \omega d|\mu| \leqslant M\|m\| .
$$

By the definitions of $\mu$ and $m_{\mu}$, the restrictions of $m$ and $m_{\mu}$ to $C_{t}$ are equal for every $t$ in $[0, \infty)$. Thus, since $U_{t \in[0, \infty)} C_{t}$ is dense in $C_{0}(\omega)$ and $m$ and $m_{\mu}$ are bounded, $m=m_{\mu}$.

We have thus shown that the map $\mu \rightarrow m_{\mu}$ is surjective and $\left\|m_{\mu}\right\| \leqslant\|\mu\| \leqslant$ $M\left\|m_{\mu}\right\|$. Therefore $M(\omega)$ and $C_{0}(\omega)^{*}$ are isomorphic Banach spaces.

Note that if $\omega$ is continuous (in the usual sense), then $M(\omega)$ and $C_{0}(\omega)^{*}$ are in fact isometric. For, if $\mu$ is in $M(\omega)$, then

$$
\begin{aligned}
\|\mu\| & =\int_{0}^{\infty} \omega d|\mu|=\sup \left\{\left|\int_{0}^{\infty} \psi \omega d \mu\right| \mid \psi \in C_{0}[0, \infty),\|\psi\|_{\infty} \leqslant 1\right\} \\
& =\sup \left\{\left|\int_{0}^{\infty} \psi d \mu\right| \| \psi \text { is continuous, }\left\|\psi \omega^{-1}\right\|_{\infty} \leqslant 1, \text { and } \lim _{t \rightarrow \infty}\left(\psi \omega^{-1}\right)(t)=0\right\} \\
& =\left\|m_{\mu}\right\| .
\end{aligned}
$$

However, $M(\omega)$ and $C_{0}(\omega)^{*}$ may not be isometric if $\omega$ is not continuous. For example, put

$$
\omega(t)= \begin{cases}2-t, & 0 \leqslant t<1 \\ 3-t, & 1 \leqslant t<2 \\ 1, & t \geqslant 2\end{cases}
$$

and let $\mu$ be the point mass at 1 . Then $\|\mu\|=2$ but $\left\|m_{\mu}\right\|=1$.

For $f$ in $L^{1}(\omega)$ and $\psi$ in $C_{0}(\omega)$ define their convolution $\psi * f$ by

$$
(\psi * f)(t)=\int_{0}^{\infty} \psi(s+t) f(s) d s
$$

Then it is not difficult to show that $\psi * f$ is in $C_{0}(\omega)$ and $\|\psi * f\| \leqslant\|\psi\|\|f\|$. It is also easily verified that $m_{f * g}(\psi)=m_{g}(\psi * f),\left(f, g \in L^{1}(\omega), \psi \in C_{0}(\omega)\right)$.

As is remarked in [1], the sequence $\left(n \chi_{[0,1 / n]}\right)$ is a bounded approximate identity for $L^{1}(\omega)$ because $\omega$ is bounded near zero. It may be shown that this sequence is also a bounded approximate identity for the Banach $L^{1}(\omega)$-bimodule $C_{0}(\omega)$. 
The required isomorphism may now be proved just as in [9]. A result analogous to this for weighted convolution algebras on locally compact groups is proved in [6].

1.3 THEOREM. Let $\omega$ be a weight function. Then $\mathscr{M}\left(L^{1}(\omega)\right)$ is isomorphic to $M(\omega)$.

As for group algebras, we will identify $l^{1}(\omega)$ with the discrete measures in $M(\omega)$ and will identify $L^{1}(\omega)$ with the measures in $M(\omega)$ which are absolutely continuous with respect to Lebesgue measure.

The notions of left, right and two-sided Banach module over a Banach algebra $A$ will be as defined in [2]. Since $L^{1}(G)$ (respectively $\left.L^{1}(\omega)\right)$ is a closed, two-sided ideal in $M(G)(M(\omega))$ and $l^{1}(G)\left(l^{1}(\omega)\right)$ is a subalgebra of $M(G)(M(\omega))$ it is clear that $L^{1}(G)\left(L^{1}(\omega)\right)$ is a two-sided Banach module over $l^{1}(G)\left(l^{1}(\omega)\right)$. The definitions of derivations and module homomorphisms wil be as given in [2].

Let $T: X \rightarrow Y$ be a linear operator, where $X$ and $Y$ are Banach spaces. Then the separating space of $T$, denoted $\mathbf{S}(T)$, is defined by

$$
\mathbf{S}(T)=\left\{y \in Y \mid \exists\left(x_{n}\right) \in X \text { with } x_{n} \rightarrow 0 \text { and } T x_{n} \rightarrow y\right\} .
$$

The properties of the separating space which we will require are given in [16]. In partcular, $\mathbf{S}(T)$ is a closed subspace of $Y$ and $\mathbf{S}(T)=(0)$ if and only if $T$ is continuous. We will also require the following lemma $[16,1.6]$.

1.4 Lemma. Let $X$ and $Y$ be Banach spaces and let $\left(S_{n}\right)$ and $\left(R_{n}\right)$ be sequences of continuous linear operators on $X$ and $Y$ respectively. If $T$ is a linear operator from $X$ into $Y$ with $T S_{n}-R_{n} T$ continuous for every $n$, then there is an integer $N$ such that $\left(R_{1} \cdots R_{n} \mathrm{~S}(T)\right)^{-}=\left(R_{1} \cdots R_{N} \mathrm{~S}(T)\right)^{-}$for all $n \geqslant N$.

\section{Some automatic continuity problems.}

The following two problems are raised in [3] as questions 22 and 24 respectively.

(A) For which locally compact groups $G$ is every derivation from $L^{1}(G)$ to a Banach $L^{1}(G)$-bimodule continuous?

(B) For which weight functions $\omega$ is every derivation from $L^{1}(\omega)$ to a Banach $L^{1}(\omega)$-bimodule continuous?

As is remarked in [3], if $G$ is an abelian group, then the closed ideals of $L^{1}(G)$ satisfy the following two conditions:

(i) if $I$ is a closed ideal with infinite codimension in $L^{1}(G)$, then there are sequences $\left(a_{n}\right),\left(b_{n}\right)$ in $L^{1}(G)$ such that $b_{n} a_{1} a_{2} \cdots a_{n} \notin I$, but $b_{n} a_{1} a_{2} \cdots$ $a_{n} a_{n+1} \in I$, for $n=1,2,3, \ldots$; 
(ii) if $I$ is a closed ideal with finite codimension in $L^{1}(G)$, then $I$ has a bounded approximate identity.

These conditions guarantee that every derivation from $L^{1}(G)$ is continuous by Theorem 2 of [11]. The fact that commutative group algebras satisfy condition (i) will be required in Section 4. It may be deduced from Wiener's Tauberian theorem $[14,7.2 .4]$.

It will be shown in Section 4 that questions $A$ and $B$ are related to two other problems.

(C) For which locally compact groups $G$ is every $l^{1}(G)$-module homomorphism from $L^{1}(G)$ to a Banach left (or two-sided) $l^{1}(G)$-module continuous?

(D) For which weight functions $\omega$ is every $l^{1}(\omega)$-module homomorphism from $L^{1}(\omega)$ to a Banach $l^{1}(\omega)$-module continuous?

Once again, if $G$ is an abelian group, then every $l^{1}(G)$-module homomorphism from $L^{1}(G)$ is continuous. In [19] it will be shown that all derivations and $l^{1}(G)$-module homomorphisms from $L^{1}(G)$ are continuous for $G$ belonging to a much wider class of groups. There are no weight functions $\omega$ for which it is known that every derivation or every $l^{1}(\omega)$-module homomorphism from $L^{1}(\omega)$ is continuous.

If $G$ is a discrete group, then $l^{1}(G)$ is equal to $L^{1}(G)$ and has a unit. Hence, in this case, $L^{1}(G)$ is a singly generated left $l^{1}(G)$-module and it is clear that every left $l^{1}(G)$-module homomorphism is continuous. The next lemma shows that, for nondiscrete $G, L^{1}(G)$ is an "approximately singly generated" left $l^{1}(G)$-module. The lemma will be required for the proofs of the automatic continuity results in [19]. It may be that there is a complete solution to (C) based on this lemma which generalizes the argument in the discrete case.

2.1 Lemma. Let $G$ be a locally compact group. Let $\left\{F_{1}, F_{2}, \ldots, F_{n}\right\}$ be a finite subset of $L^{1}(G), U$ be a neighborhood of $e$, and $\varepsilon>0$. Then there is an $F$ in $L^{1}(G)$ and $\left\{f_{1}, f_{1}, \ldots, f_{n}\right\} \subseteq l^{1}(G)$ with $\operatorname{supp}(F) \subseteq U ;\|F\|=1 ;\left\|f_{k}\right\| \leqslant\left\|F_{k}\right\|, \quad k=$ $1,2, \ldots, n$; and $\left\|F_{k}-f_{k} * F\right\|<\varepsilon, k=1,2, \ldots, n$.

Proof. Suppose first of all that the $F_{j}$ 's are positive, continuous functions with compact support in $G$. Then the result follows from the proof of the existence and uniqueness of Haar measure in [9, Theorem 15.5]. The $f_{j}$ 's and $F$ are constructed in the lemma of step III of that proof.

Next suppose that the $F_{j}$ 's are characteristic functions of open sets. Then the result holds because characteristic functions may be approximated, in the $L^{1}$-norm, by positive, continuous functions with compact support. Hence the result holds for simple functions, that is, for functions which are linear combination of characteristic functions. 
Finally, the result holds in general because the simple functions are dense in $L^{1}(G)$.

Next we have the corresponding lemma for $L^{1}(\omega)$. Its proof is straightforward and will be omitted.

2.2 Lemma. Let $\omega$ be a weight function which is bounded near zero by $M$. Let $\left\{F_{1}, F_{2}, \ldots, F_{n}\right\}$ be a finite subset of $L^{1}(\omega)$ and $\varepsilon, \delta>0$. Then there is an $F$ in $L^{1}(\omega)$ and $\left\{f_{1}, f_{2}, \ldots, f_{n}\right\} \subseteq l^{1}(\omega)$ with $\operatorname{supp}(F) \subseteq[0, \delta] ;\|F\| \leqslant M ;\left\|f_{k}\right\| \leqslant\left\|F_{k}\right\|$, $k=1,2, \ldots, n$; and $\left\|F_{k}-f_{k} * F\right\|<\varepsilon, k=1,2, \ldots, n$.

Now approximate identity arguments applying Cohen's Factorization Theorem $[2,11.10]$ have been widely used in the proofs of automatic continuity results (for example, see [11, 12]). Thus an obvious question is whether a factorization theorem can be deduced from 2.1 and 2.2. We ask whether the following assertion holds for each nondiscrete group $G$ and each weight function $\omega$.

(E) There is an integer $k$ such that, for each sequence $\left(F_{n}\right)_{n=1}^{\infty}$ converging to zero in $L^{1}(G)\left(L^{1}(\omega)\right)$, there are functions $F^{1}, F^{2}, \ldots, F^{k}$ in $L^{1}(G)\left(L^{1}(\omega)\right)$ and sequences $\left(f_{n}^{1}\right)_{n=1}^{\infty},\left(f_{n}^{2}\right)_{n=1}^{\infty}, \ldots,\left(f_{n}^{k}\right)_{n=1}^{\infty}$ converging to zero in $l^{1}(G)\left(l^{1}(\omega)\right)$ with

$$
F_{n}=\sum_{j=1}^{k} f_{n}^{j} * F^{j}, \quad n=1,2,3, \ldots
$$

It is immediate that, if (E) holds for some $G$, then every left $l^{1}(G)$-module homomorphism from $L^{1}(G)$ is continuous and similarly for weight functions.

Since Lemmas 2.1 and 2.2 show that $L^{1}(G)$ and $L^{1}(\omega)$ are "approximately singly generated" it is to be expected that, if $(E)$ holds at all, then it will hold with $k=1$. However, the example below shows that, in the group algebra case, (E) may not hold with $k=1$.

2.3 EXAMPLE. Let $\mathbf{T}$ be the circle group with Haar measure normalized so that $\lambda(\mathbf{T})=1$. Let $\left\{\chi_{n} \mid n \in \mathbf{Z}\right\}$ be the set of continuous characters on $\mathbf{T}$ and define $F_{n}=\left(1 / 2 n^{2}\right) \sum_{|k|<n} \chi_{k}, n=1,2,3, \ldots$ Then each $F_{n}$ is in $L^{1}(\mathbf{T})$ and

$$
\left\|F_{n}\right\| \leqslant \frac{1}{2 n^{2}} \sum_{|k|<n}\left\|\chi_{n}\right\|<\frac{1}{n} .
$$

Hence $\left(F_{n}\right)_{n=1}^{\infty}$ is a sequence converging to zero in $L^{1}(\mathbf{T})$.

Suppose that there are functions $F$ in $L^{1}(\mathbf{T})$ and $f_{n}$ in $l^{1}(\mathbf{T}), n=1,2,3, \ldots$, such that $F_{n}=f_{n} * F, n=1,2,3, \ldots$ Taking Fourier transforms we have

$$
\hat{F}_{n}(k)=\hat{f}_{n}(k) \hat{F}(k), \quad n=1,2,3, \ldots, \quad k \in \mathbf{Z} .
$$

If $\hat{F}(k)=0$ for some $k$, then $\hat{F}_{n}(k)=0$, for every $n$. Thus, since $\hat{F}_{n}(k)=1 / 2 n^{2}$ when $n>|k|$, we must have that $\hat{F}(k) \neq 0$ for every $k \in \mathbf{Z}$. Since $\hat{F}_{n}(k)=0$ for 
$|k| \geqslant n$, it now follows that $\hat{f}_{n}(k)=0$ for $|k| \geqslant n, n=1,2,3, \ldots$ Hence, $f_{n}$ is in the span of $\left\{\chi_{-n+1}, \chi_{-n+2}, \ldots, \chi_{n-1}, \chi_{n-1}\right\}$, which contradicts the assumption that $f_{n}$ is in $l^{1}(\mathbf{T})$.

\section{Some automatic continuity lemmas}

The results of this section are based on some lemmas in Chapters 4 and 5 of [17]. They simplify the problems of continuity of derivations and module homomorphisms from $L^{1}(G)$ (and $L^{1}(\omega)$ ) by reducing them to the special cases when the range module is $L^{\infty}(G \times G)$ or $l^{\infty}(G)\left(\left(L^{1}(\omega) \hat{\otimes} L^{1}(\omega)\right)^{*}\right.$ or $\left.l^{\infty}(\omega)\right)$.

Let $A$ be a Banach algebra and $A \hat{\otimes} A$ be the projective tensor product of $A$ with itself (see [2, 42.9]). Define $A \hat{\otimes} A$ to be a Banach $A$-bimodule by putting $f \cdot(a \otimes b)=(f a) \otimes b$ and $(a \otimes b) \cdot f=a \otimes(b f),(f \in A, a \otimes b \in A \hat{\otimes} A)$, and then extending this action of $f$ to the rest of $A \hat{\otimes} A$ by linearity and continuity. The dual space, $(A \hat{\otimes} A)^{*}$, then becomes a Banach $A$-bimodule under the dual action. If $X$ is a left $A$-module, then $A \cdot X$ will denote $\{a \cdot x \mid a \in A ; x \in X\}$ and, if $X$ is an $A$-bimodule, then $A \cdot X \cdot A$ will denote $\{a \cdot x \cdot b \mid a, b \in A$; $x \in X\}$.

The next lemma was suggested to me by Professor B. E. Johnson.

3.1 LemMA. Let $A$ be a Banach algebra with a two-sided bounded approximate identity.

(i) If $X$ is a Banach A-bimodule and $D: A \rightarrow X$ is a derivation, then $\mathbf{S}(D)$ is a closed submodule of $X$ and $\mathbf{S}(D)=A \cdot \mathbf{S}(D) \cdot A$.

(ii) If every derivation from $A$ to $(A \hat{\otimes} A)^{*}$ is continuous, then every derivation from $A$ to an arbitrary Banach $A$-bimodule $X$ is continuous.

Proof. (i) It is well known and easily verified that, when $D$ is a derivation, $\mathbf{S}(D)$ is a closed submodule of $X$.

Now let $s$ be in $\mathbf{S}(D)$ and $\left(a_{n}\right)$ be a sequence in $A$ with $a_{n} \rightarrow 0$ and $D\left(a_{n}\right) \rightarrow s$. Since $A$ has a bounded approximate identity there is, by Cohen's Factorization Theorem, an $a$ in $A$ and a sequence $\left(a_{n}^{\prime}\right)$ in $A$ such that $a_{n}^{\prime} \rightarrow 0$ and $a_{n}=a a_{n}^{\prime}$ for every $n$. Hence,

$$
s=\lim _{n \rightarrow \infty} D\left(a a_{n}^{\prime}\right)=\lim _{n \rightarrow \infty}\left(a \cdot D\left(a_{n}^{\prime}\right)+D(a) \cdot a_{n}^{\prime}\right)=\lim _{n \rightarrow \infty}\left(a \cdot D\left(a_{n}^{\prime}\right)\right) .
$$

Take $\varepsilon>0$ and choose an integer $n$ such that $\left\|s-a \cdot D\left(a_{n}^{\prime}\right)\right\|<\varepsilon$. Let $\left(e_{\mu}\right)_{\mu \in N}$ be a bounded approximate identity for $A$ with bound $K$ and choose $\mu(n)$ such that

$$
\left\|a-e_{\mu} a\right\|<\varepsilon /\left\|D\left(a_{n}^{\prime}\right)\right\| \quad(\mu \geqslant \mu(n)) .
$$


Then, for every $\mu \geqslant \mu(n)$,

$$
\begin{aligned}
\left\|s-e_{\mu} \cdot s\right\| & \leqslant\left\|s-a \cdot D\left(a_{n}^{\prime}\right)\right\|+\left\|\left(a-e_{\mu} a\right) \cdot D\left(a_{n}^{\prime}\right)\right\| \\
& +\left\|e_{\mu} \cdot\left(a \cdot D\left(a_{n}^{\prime}\right)-s\right)\right\|<\varepsilon(1+1+K) .
\end{aligned}
$$

Hence $\left(e_{\mu}\right)_{\mu \in N}$ is a bounded approximate identity for $\mathbf{S}(D)$ regarded as a left Banach $A$-module.

Similarly, $\left(e_{\mu}\right)_{\mu \in N}$ is a bounded approximate identity for $\mathbf{S}(D)$ as a right Banach $A$-module. Therefore, for each $s$ in $\mathbf{S}(D)$, there is, by Cohen's Factorization Theorem, an $s^{\prime}$ in $\mathbf{S}(D)$ and $c, d$ in $A$ such that $s=c \cdot s^{\prime} \cdot d$.

(ii) Suppose that all derivations from $A$ to $(A \hat{\otimes} A)^{*}$ are continuous. Let $D$ be a derivation from $A$ to $X$ and let $s$ be in $\mathbf{S}(D)$. Then, by (i), there is an $s^{\prime}$ in $\mathrm{S}(D)$ and $c, d$ in $A$ with $s=c \cdot s^{\prime} \cdot d$.

Choose a linear functional, $f$, in $X^{*}$ such that $f(s)=\|s\|$ and define a linear operator, $R_{f}$, from $X$ to $(A \hat{\otimes} A)^{*}$ by

$$
\left(R_{f} x\right)(a \otimes b)=f(b \cdot x \cdot a) \quad(x \in X, a \otimes b \in A \hat{\otimes} A) .
$$

Let $x$ be in $X$ and $t=\sum_{i=1}^{n} a_{i} \otimes b_{i}$ be in $A \hat{\otimes} A$. By the definition of the norm on $A \hat{\otimes} A$ we may, for each $\varepsilon>0$, suppose that $\sum_{i=1}^{n}\left\|a_{i}\right\|\left\|b_{i}\right\|<\|t\|+\varepsilon$. Then

$$
\left|\left(R_{f} x\right)(t)\right|=\left|\sum_{i=1}^{n} f\left(b_{i} \cdot x \cdot a_{i}\right)\right| \leqslant\|f\|\|x\|\left(\sum_{i=1}^{n}\left\|a_{i}\right\|\left\|b_{i}\right\|\right)<\|f\|\|x\|(\|t\|+\varepsilon) .
$$

Hence $R_{f}$ is a bounded linear operator and $\left\|R_{f}\right\| \leqslant\|f\|$.

Now let $a, b$ and $c$ be in $A$ and $x$ be in $X$. Then

$$
\begin{aligned}
\left(R_{f}(x \cdot c)\right)(a \otimes b) & =f(b \cdot(x \cdot c) \cdot a)=f(b \cdot(x \cdot c) \cdot a)=f(b \cdot x \cdot(c a)) \\
& =\left(R_{f} x\right)(c \cdot a \otimes b)=\left(\left(R_{f} x\right) \cdot c\right)(a \otimes b) .
\end{aligned}
$$

Hence $R_{f}(x \cdot c)=\left(R_{f} x\right) \cdot c$. Similarly, $R_{f}(c \cdot x)=c \cdot\left(R_{f} x\right)$. Therefore $R_{f}$ is a bimodule homomorphism from $X$ to $(A \hat{\otimes} A)^{*}$. It follows that $R_{f} \circ D$ is a derivation from $A$ to $(A \hat{\otimes} A)^{*}$ and is thus continuous.

Since $s^{\prime}$ is in $\mathbf{S}(D)$ there is a sequence $\left(a_{n}\right)$ in $A$ such that $a_{n} \rightarrow 0$ and $D\left(a_{n}\right) \rightarrow s^{\prime}$. Hence, since $R_{f} \circ D$ is continuous,

$$
\begin{aligned}
0 & =\lim _{n \rightarrow \infty}\left(R_{f} \circ D\right)\left(a_{n}\right)(d \otimes c) \\
& =\lim _{n \rightarrow \infty} R_{f}\left(D\left(a_{n}\right)\right)(d \otimes c) \\
& =\lim _{n \rightarrow \infty} f\left(c \cdot D\left(a_{n}\right) \cdot d\right) \\
& =f\left(c \cdot s^{\prime} \cdot d\right) \\
& =f(s)=\|s\| .
\end{aligned}
$$

Therefore $s=0$. It follows that $\mathbf{S}(D)=(0)$ and so $D$ is continuous. 
The same argument, slightly simplified, may be used to prove Lemma 3.2 below.

3.2 LEMMA. Let $A$ be a Banach algebra with a two-sided bounded approximate identity and $X$ be a Banach left $A$-module such that $A$ has a bounded approximate identity for $X$.

(i) If $Y$ is a Banach left $A$-module and $T: X \rightarrow Y$ is an A-module homomorphism, then $\mathbf{S}(T)$ is a closed submodule of $Y$ and $\mathbf{S}(T)=A \cdot \mathbf{S}(T)$.

(ii) If every $A$-module homomorphism from $X$ to $A^{*}$ (regarded as a left $A$-module under the dual action) is continuous, then every A-module homomorphism from $X$ to an arbitrary Banach left $A$-module $Y$ is continuous.

The following corollary was first proved in [12]. It is required for the proof of the next lemma.

3.3 Corollary. Let $A$ be a Banach algebra with a two-sided bounded approximate identity. Then every homomorphism from $A$ to a Banach right $A$-module $X$ is continuous.

Proof. Let $T$ be a such a homomorphism. If we make $X$ into an $A$-bimodule by defining $a \cdot x=0(a \in A, x \in X)$, then $T$ becomes a derivation. Hence, by 3.1(i), $\mathbf{S}(T)=A \cdot \mathbf{S}(T) \cdot A=(0)$.

The next few lemmas will be concerned only with derivations from $L^{1}(G)$ or $L^{1}(\omega)$. It will be necessary for $\left(L^{1}(G) \hat{\otimes} L^{1}(G)\right)^{*}$ to be an $M(G)$-bimodule and $\left(L^{1}(\omega) \hat{\otimes} L^{1}(\omega)\right)^{*}$ to be an $M(\omega)$-bimodule. For this, recall that $L^{1}(G)$ is a closed two-sided ideal in $M(G)$ and so may be regarded as an $M(G)$-bimodule. We may now define $L^{1}(G) \hat{\otimes} L^{1}(G)$ to be a Banach $M(G)$-bimodule by putting $\mu \cdot(a \otimes b)=(\mu * a) \otimes b$ and $(a \otimes b) \cdot \mu=a \otimes(b * \mu), \quad\left(a, b \in L^{1}(G), \quad \mu \in\right.$ $M(G)$ ), and extending this action of $M(G)$ to the rest of $L^{1}(G) \hat{\otimes} L^{1}(G)$ by linearity and continuity. The action of $M(G)$ on $\left(L^{1}(G) \hat{\otimes} L^{1}(G)\right)^{*}$ is now defined to be the dual module action. We define $\left(L^{1}(\omega) \hat{\otimes} L^{1}(\omega)\right)^{*}$ to be a Banach $M(\omega)$-bimodule in the same way.

3.4 LEMMA. Let $G$ be a locally compact group and $D$ be a derivation from $L^{1}(G)$ to $\left(L^{1}(G) \hat{\otimes} L^{1}(G)\right)^{*}$. Then there is a unique derivation, $\bar{D}$, from $M(G)$ to $\left(L^{1}(G) \hat{\otimes} L^{1}(G)\right)^{*}$ which extends $D$.

Proof. For each $\mu$ in $M(G)$ define the operator $S_{\mu}$ from $L^{1}(G)$ to $\left(L^{1}(G) \hat{\otimes}\right.$ $\left.L^{1}(G)\right)^{*}$ by $S_{\mu}(f)=D(\mu * f)-\mu \cdot D(f)\left(f \in L^{1}(G)\right)$. Then, for $f$ and $g$ in 
$L^{1}(G)$,

$$
\begin{aligned}
S_{\mu}(f * g)= & D(\mu * f * g)-\mu \cdot D(f * g) \\
= & (\mu * f) \cdot D(g)+D(\mu * f) \cdot g \\
& -(\mu * f) \cdot D(g)-\mu \cdot D(f) \cdot g \\
= & S_{\mu}(f) \cdot g .
\end{aligned}
$$

Hence $S_{\mu}$ is a right $L^{1}(G)$-module homomorphism from $L^{1}(G)$ to $\left(L^{1}(G) \hat{\otimes}\right.$ $\left.L^{1}(G)\right)^{*}$. Since $L^{1}(G)$ has a two-sided bounded approximate identity, it follows from 3.1 that $S_{\mu}$ is continuous.

Let $\left(e_{\lambda}\right)_{\lambda \in \Lambda}$ be a bounded approximate identity for $L^{1}(G)$. Then $\left(S_{\mu}\left(e_{\lambda}\right)\right)_{\lambda \in \Lambda}$ is a bounded net in $\left(L^{1}(G) \hat{\otimes} L^{1}(G)\right)^{*}$ because $S_{\mu}$ is continuous. For each $\mu$ in $M(G), f$ in $L^{1}(G)$ and $x$ in $L^{1}(G) \hat{\otimes} L^{1}(G)$,

$$
\begin{aligned}
S_{\mu}(f)(x) & =\lim _{\lambda} S_{\mu}\left(e_{\lambda} * f\right)(x) \quad \text { (since } S_{\mu} \text { is continuous) } \\
& =\lim _{\lambda}\left(S_{\mu}\left(e_{\lambda}\right) \cdot f\right)(x) \quad(\text { by }(3.1)) .
\end{aligned}
$$

Now $\left(e_{\lambda}\right)_{\lambda \in \Lambda}$ is a bounded approximate identity for the $L^{1}(G)$-bimodule $L^{1}(G)$ $\hat{\otimes} L^{1}(G)$, and so, by Cohen's factorization theorem, every element $x^{\prime}$ may be expressed as $x^{\prime}=f \cdot x$, for some $f$ in $L^{1}(G)$ and $x$ in $L^{1}(G) \hat{\otimes} L^{1}(G)$. Hence, by (3.2), $\lim _{\lambda} S_{\mu}\left(e_{\lambda}\right)\left(x^{\prime}\right)$ exists for every $x^{\prime}$ in $L^{1}(G) \hat{\otimes} L^{1}(G)$ and so $\left(S_{\mu}\left(e_{\lambda}\right)\right)_{\lambda \in \Lambda}$ converges in the $w^{*}$-topology on $\left(L^{1}(G) \hat{\otimes} L^{1}(G)\right)^{*}$.

Define $\bar{D}$ by putting $\bar{D}(\mu)=\lim _{\lambda} S_{\mu}\left(e_{\lambda}\right),(\mu \in M(G))$. Then, by (3.2), for each $f$ in $L^{1}(G)$ and $\mu$ in $M(G)$,

$$
\begin{aligned}
S_{\mu}(f)(x) & =\lim _{\lambda} S_{\mu}\left(e_{\lambda}\right)(f \cdot x)=\bar{D}(\mu)(f \cdot x) \\
& =(\bar{D}(\mu) \cdot f)(x), \quad\left(x \in L^{1}(G) \hat{\otimes} L^{1}(G)\right) .
\end{aligned}
$$

Thus,

$$
S_{\mu}(f)=\bar{D}(\mu) \cdot f \quad\left(f \in L^{1}(G), \mu \in M(G)\right) .
$$

To show that $D$ is a derivation, let $\mu$ and $\nu$ be in $M(G)$. Then, for every $f$ in $L^{1}(G)$

$$
\begin{aligned}
\bar{D}(\mu * \nu) \cdot f & =S_{\mu * \nu}(f) \\
& =D(\mu * \nu * f)-(\mu * \nu) \cdot D(f) \\
& =D(\mu * \nu * f)-\mu \cdot D(\nu * f)+\mu \cdot D(\nu * f)-(\mu * \nu) \cdot D(f) \\
& =S_{\mu}(\nu * f)+\mu \cdot S_{\nu}(f) \\
& =\bar{D}(\mu) \cdot(\nu * f)+\mu \cdot \bar{D}(\nu) \cdot f \\
& =(\bar{D}(\mu) \cdot \nu+\mu \cdot \bar{D}(\nu)) \cdot f
\end{aligned}
$$


Hence

$$
\begin{aligned}
\bar{D}(\mu * \nu) & =\lim _{\lambda} \bar{D}(\mu * \nu) \cdot e_{\lambda} \\
& =\lim _{\lambda}(D(\mu) \cdot \nu+\mu \cdot \bar{D}(\nu)) \cdot e_{\lambda} \\
& =\mu \cdot \bar{D}(\nu)+\bar{D}(\mu) \cdot \nu,
\end{aligned}
$$

(where the limits are with respect to the $w^{*}$-topology on $\left(L^{1}(G) \hat{\otimes} L^{1}(G)\right)^{*}$ ).

To show that $\bar{D}$ extends $D$, let $f$ be in $L^{1}(G)$. Then, for every $g$ in $L^{1}(G)$, $\bar{D}(f) \cdot g=S_{f}(g)=D(f * g)-f \cdot D(g)=D(f) \cdot g$. Hence, $\bar{D}(f)=\lim _{\lambda} \bar{D}(f)$ - $e_{\lambda}=\lim _{\lambda} D(f) \cdot e_{\lambda}=D(f)$.

Finally, let $\delta$ be a derivation from $M(G)$ to $\left(L^{1}(G) \hat{\otimes} L^{1}(G)\right)^{*}$ which extends $D$. Then, for every $\mu$ in $M(G)$,

$$
\begin{aligned}
(\bar{D}-\delta)(\mu) & =\lim _{\lambda}(\bar{D}-\delta)(\mu) \cdot e_{\lambda} \\
& =\lim _{\lambda}(\bar{D}-\delta)\left(\mu * e_{\lambda}\right)-\mu \cdot(\bar{D}-\delta)\left(e_{\lambda}\right)=0,
\end{aligned}
$$

because $\mu * e_{\lambda}$ and $e_{\lambda}$ are in $L^{1}(G)$. Therefore $\bar{D}$ is the unique derivation extending $D$.

The argument used above will also prove the next lemma.

3.5 Lemma. Let $\omega$ be a weight function and $D$ be a derivation from $L^{1}(\omega)$ to $\left(L^{1}(\omega) \hat{\otimes} L^{1}(\omega)\right)^{*}$. Then there is a unique derivation, $\bar{D}$, from $M(\omega)$ to $\left(L^{1}(\omega) \hat{\otimes}\right.$ $\left.L^{1}(\omega)\right)^{*}$ which extends $D$.

The next two lemmas begin to show the value of being able to extend derivations from $L^{1}$-algebras to measure algebras.

3.6 Lemma. Let $\bar{D}$ be a derivation from $M(G)$ to $\left(L^{1}(G) \hat{\otimes} L^{1}(G)\right)^{*}$ and define a map $\delta$ from $G$ to $\left(L^{1}(G) \hat{\otimes} L^{1}(G)\right)^{*}$ by $\delta(x)=\bar{D}(\bar{x}),(x \in G)$. Suppose that there is a neighborhood $U$ of the identity in $G$ and a positive constant $M$ such that $\|\delta(x)\| \leqslant M,(x \in U)$. Then $\delta$ is continuous with respect to the group topology on $G$ and the $w^{*}$-topology on $\left(L^{1}(G) \hat{\otimes} L^{1}(G)\right)^{*}$.

Proof. Let $\left(x_{\lambda}\right)_{\lambda \in \Lambda}$ be a net in $G$ which converges to $e$, the identity element of $G$. Then, by passing to a subnet if necessary, we may suppose that $\left\|\delta\left(x_{\lambda}\right)\right\| \leqslant M$, $(\lambda \in \Lambda)$. Now the unit ball in $\left(L^{1}(G) \hat{\otimes} L^{1}(G)\right)^{*}$ is compact in the $w^{*}$-topology [9, Theorem (B.25)]. Hence $\left(\delta\left(x_{\lambda}\right)\right)_{\lambda \in \Lambda}$ has an accumulation point. Let $X$ be such an accumulation point and $\left(\delta\left(x_{\mu}\right)\right)_{\mu \in N}$ be a subnet which converges to $X$ in the $w^{*}$-topology. 
For each $f$ in $L^{1}(G) \hat{\otimes} L^{1}(G)$ and each $\mu$ in $N$,

$$
\begin{aligned}
\delta\left(x_{\mu}^{2}\right)(f)= & {\left[\bar{x}_{\mu} \cdot \bar{D}\left(\bar{x}_{\mu}\right)+\bar{D}\left(\bar{x}_{\mu}\right) \cdot \bar{x}_{\mu}\right](f) } \\
= & \bar{D}\left(\bar{x}_{\mu}\right)\left(\bar{x}_{\mu} \cdot f+f \cdot \bar{x}_{\mu}\right) \\
= & 2 \delta\left(x_{\mu}\right)(f)+\delta\left(x_{\mu}\right)\left(\bar{x}_{\mu} \cdot f-f\right) \\
& +\delta\left(x_{\mu}\right)\left(f \cdot \bar{x}_{\mu}-f\right) .
\end{aligned}
$$

Now

$$
\begin{aligned}
\left|\delta\left(x_{\mu}\right)\left(\bar{x}_{\mu} \cdot f-f\right)\right| & \leqslant\left\|\delta\left(x_{\mu}\right)\right\|\left\|\bar{x}_{\mu} \cdot f-f\right\| \\
& \leqslant M\left\|\bar{x}_{\mu} \cdot f-f\right\| \stackrel{\mu}{\rightarrow} 0,
\end{aligned}
$$

because left translation in $L^{1}(G) \hat{\otimes} L^{1}(G)$ is strongly continuous. Similarly, $\delta\left(x_{\mu}\right)\left(f \cdot \bar{x}_{\mu}-f\right) \stackrel{\mu}{\rightarrow} 0$. Hence, $\lim _{\mu} \delta\left(x_{\mu}^{2}\right)(f)=2 \lim _{\mu} \delta\left(x_{\mu}\right)(f)=2 X(f)$, for every $f$ in $L^{1}(G) \hat{\otimes} L^{1}(G)$. That is, $\left(\delta\left(x_{\mu}^{2}\right)\right)_{\mu \in N}$ converges to $2 X$ in the $w^{*}$-topology. It may be shown in the same way that, for every positive integer $k$, $\lim _{\mu} \delta\left(x_{\mu}^{k}\right)=k X$.

Suppose that $X \neq 0$ and choose an integer $k$ such that $k\|X\|>M$. Then $\delta\left(x_{\mu}^{k}\right) \rightarrow k X$, but eventually $\left\|\delta\left(x_{\mu}^{k}\right)\right\| \leqslant M<\|k X\|$, which is a contradiction. Hence $X=0$ and 0 is the only accumulation point of $\left(\delta\left(x_{\lambda}\right)\right)_{\lambda \in \Lambda}$. Therefore, $\delta\left(x_{\lambda}\right) \stackrel{w^{*}}{\rightarrow} 0$ whenever $\left(x_{\lambda}\right)_{\lambda \in \Lambda}$ is a net in $G$ which converges to $e$.

Now, let $x$ be in $G$ and $\left(x_{\lambda}\right)_{\lambda \in \Lambda}$ be a net which converges to $x$. Then $x^{-1} x_{\lambda} \rightarrow e$, and so,

$$
\delta\left(x_{\lambda}\right)=\bar{D}\left(\bar{x} *\left(x^{-1} x_{\lambda}\right)^{-}\right)=\bar{x} \cdot \delta\left(x^{-1} x_{\lambda}\right)+\delta(x) \cdot\left(x^{-1} x_{\lambda}\right)^{-\stackrel{w^{*}}{\rightarrow} \delta(x) .}
$$

Therefore $\delta$ is continuous.

3.7 LemMa. Let $\bar{D}$ be a derivation from $M(\omega)$ to $\left(L^{1}(\omega) \hat{\otimes} L^{1}(\omega)\right)^{*}$ and define a map $\delta$ from $[0, \infty)$ to $\left(L^{1}(\omega) \hat{\otimes} L^{1}(\omega)\right)^{*}$ by $\delta(x)=\bar{D}(\bar{x}),(x \in[0, \infty))$. Suppose that there is an $\varepsilon>0$ and a positive constant $M$ such that $\|\delta(x)\| \leqslant M,(x \in[0, \varepsilon))$. Then $\delta$ is continuous with respect to the usual topology on $[0, \infty)$ and the $w^{*}$-topology on $\left(L^{1}(\omega) \hat{\otimes} L^{1}(\omega)\right)^{*}$.

Proof. It may be shown in exactly the same way as in (3.6) that, if $\left(x_{n}\right)$ is a sequence in $[0, \infty)$ which converges to 0 , then $\delta\left(x_{n}\right) \stackrel{w^{*}}{\rightarrow} 0$.

Let $x$ be any number in $[0, \infty)$ and $\left(x_{n}\right)$ be a sequence converging to $x$ with $x_{n} \geqslant x$ for every $n$. Then we must have that for every $n, x_{n}=x+t_{n}$, where $t_{n} \in[0, \infty)$ and $t_{n} \rightarrow 0$. Hence

$$
\delta\left(x_{n}\right)=\bar{D}\left(\bar{x} * \bar{t}_{n}\right)=x \cdot \delta\left(t_{n}\right)+\delta(x) \cdot \bar{t}_{n} \stackrel{w^{*}}{\rightarrow} \delta(x) .
$$


Now let $\left(x_{n}\right)$ be a sequence converging to $x$ with $x_{n} \leqslant x$ for every $n$. Then $\left\|\delta\left(x_{n}\right)\right\|$ is bounded. To show this, choose $y<x$ with $|y-x|<\varepsilon$. Then $x_{n} \in$ $[y, x]$ for all but finitely many $n$ and so

$$
\left\|\delta\left(x_{n}\right)\right\| \leqslant\left\|\bar{y} \cdot \delta\left(x_{n}-y\right)\right\|+\left\|\delta(y) \cdot\left(x_{n}-y\right)^{-}\right\|,
$$

which is bounded because $x_{n}-y$ is in $[0, \varepsilon)$ and so $\left\|\delta\left(x_{n}-y\right)\right\| \leqslant M$. We have that for every $n, x=x_{n}+t_{n}$, where $t_{n} \in[0, \infty)$ and $t_{n} \rightarrow 0$. Hence, for each $F$ in $L^{1}(\omega) \hat{\otimes} L^{1}(\omega)$,

$$
\begin{aligned}
& \left|\left(\delta(x)-\delta\left(x_{n}\right)\right)(F)\right|=\left|\delta\left(x_{n}\right)\left(\bar{t}_{n} \cdot F-F\right)+\delta\left(t_{n}\right)\left(F \cdot \bar{x}_{n}\right)\right| \\
& \quad \leqslant\left\|\delta\left(x_{n}\right)\right\|\left\|\bar{t}_{n} \cdot F-F\right\|+\left\|\delta\left(t_{n}\right)\right\|\left\|F \cdot \bar{x}_{n}-F \cdot \bar{x}\right\|+\left|\delta\left(t_{n}\right)(F \cdot \bar{x})\right| \rightarrow 0 .
\end{aligned}
$$

Therefore, $\delta$ is continuous.

\section{Applications of the lemmas}

We will now use the lemmas of the previous section to show how the continuity of derivations and module homomorphisms problems may be related. For the first result, let $\mathbb{F}_{0}$ denote the free group on a countably infinite number of generators.

4.1 THEOREM. Suppose that there is a discontinuous derivation from $L^{1}(G)$ to a Banach $L^{1}(G)$-bimodule $X$ for some locally compact group $G$. Then either there is a discontinuous $l^{1}(G)$-bimodule homomorphism from $L^{1}(G)$ to $\left(L^{1}(G) \hat{\otimes} L^{1}(G)\right)^{*}$ or there is a discontinuous derivation from $l^{1}\left(\mathbb{F}_{0}\right)$ to $\left(l^{1}\left(\mathbb{F}_{0}\right) \hat{\otimes} l^{1}\left(\mathbb{F}_{0}\right)\right)^{*}$.

Proof. By Lemma 3.1(ii), there is a discontinuous derivation $D$ from $L^{1}(G)$ to $\left(L^{1}(G) \hat{\otimes} L^{1}(G)\right)^{*}$ and, by Lemma 3.4 , this extends to give a derivation $\bar{D}$ from $M(G)$ to $\left(L^{1}(G) \hat{\otimes} L^{1}(G)\right)^{*}$.

Suppose that the restriction of $\bar{D}$ to $l^{1}(G)$ (the subalgebra of discrete measures in $M(G))$ is continuous. Then $\{\bar{D}(\bar{x}) \mid x \in G\}$ is a bounded set in $\left(L^{1}(G) \hat{\otimes}\right.$ $\left.L^{1}(G)\right)^{*}$ and so by Lemma 3.6 the map $\delta: x \mapsto \bar{D}(\bar{x})$ is continuous with respect to the group topology on $G$ and the $w^{*}$-topology on $\left(L^{1}(G) \hat{\otimes} L^{1}(G)\right)^{*}$.

Define a map $\Delta: M(G) \rightarrow\left(L^{1}(G) \hat{\otimes} L^{1}(G)\right)^{*}$ by

$$
(\Delta(\mu))(\psi)=\int_{G} \bar{D}(\bar{x})(\psi) d \mu(x) \quad\left(\mu \in M(G), \psi \in L^{1}(G) \hat{\otimes} L^{1}(G)\right) .
$$

The integral exists because the function $x \mapsto \bar{D}(\bar{x})(\psi)$ is bounded and continuous on $G$. It is clear that $\Delta$ is a bounded linear operator and that the restrictions of $\Delta$ and $\bar{D}$ to $l^{\mathrm{l}}(G)$ are equal. 
Let $\mu$ and $\nu$ be in $M(G)$. Then, for every $\psi$ in $L^{1}(G) \hat{\otimes} L^{1}(G)$,

$$
\begin{aligned}
(\Delta(\mu * \nu))(\psi) & =\int_{G} \bar{D}(\bar{x})(\psi) d(\mu * \nu)(x) \\
& =\int_{G \times G} \bar{D}(\overline{x y})(\psi) d \mu(x) d \nu(y) \quad \text { (by [9, Theorem 19.10]) } \\
& =\int_{G \times G}(\bar{x} \cdot \bar{D}(\bar{y})+\bar{D}(\bar{x}) \cdot \bar{y})(\psi) d \mu(x) d \nu(y) \\
& =\int_{G \times G}[\bar{D}(\bar{y})(\psi \cdot \bar{x})+\bar{D}(\bar{x})(\bar{y} \cdot \psi)] d \mu(x) d \nu(y) \\
& =\int_{G} \bar{D}(\bar{y})(\psi \cdot \mu) d \nu(y)+\int_{G} \bar{D}(\bar{x})(\nu \cdot \psi) d \mu(x) \\
& =\Delta(\nu)(\psi \cdot \mu)+\Delta(\mu)(\nu \cdot \psi) \\
& =(\mu \cdot \Delta(\nu)+\Delta(\mu) \cdot \nu)(\psi) .
\end{aligned}
$$

Hence, $\Delta$ is a derivation from $M(G)$ to $\left(L^{1}(G) \hat{\otimes} L^{1}(G)\right)^{*}$.

Now let $f$ be in $l^{1}(G)$ and $g$ be in $L^{1}(G)$. Then,

$$
\begin{aligned}
(\bar{D}-\Delta)(f * g) & =f \cdot(\bar{D}-\Delta)(g)+(\bar{D}-\Delta)(f) \cdot g \\
& =f \cdot(\bar{D}-\Delta)(g),
\end{aligned}
$$

because $\bar{D}$ and $\Delta$ agree on $l^{1}(G)$. Similarly, $(\bar{D}-\Delta)(g * f)=(\bar{D}-\Delta)(g) \cdot f$. Hence the restriction of $\bar{D}-\Delta$ to $L^{1}(G)$ is a $l^{1}(G)$-bimodule homomorphism to $\left(L^{1}(G) \hat{\otimes} L^{1}(G)\right)^{*}$. Since the restriction of $\bar{D}$ to $L^{1}(G)$ is $D$, which is not continuous, and since $\Delta$ is continuous, this restriction of $\bar{D}-\Delta$ to $L^{1}(G)$ is not continuous.

On the other hand, suppose that the restriction of $\bar{D}$ to $l^{1}(G)$ is not continuous. Then there is a sequence $\left(f_{n}\right)$ in $l^{1}(G)$ such that $f_{n} \rightarrow 0$ but $\bar{D}\left(f_{n}\right) \nrightarrow 0$. Let $H$ be the discrete subgroup of $G$ which is generated by the supports of the functions $f_{1}, f_{2}, \ldots$ Then $H$ is countably generated and the restriction of $\bar{D}$ to $l^{1}(H)$ is not continuous.

Since $H$ is countably generated it is a quotient of $\mathbb{F}_{0}$ and so there is a Banach algebra homomorphism $A$ from $l^{1}\left(\mathbb{F}_{0}\right)$ onto $l^{1}(H)$. Then $\bar{D} \circ A$ is a discontinuous derivation from $l^{1}\left(\mathbb{F}_{0}\right)$ to $\left(L^{1}(G) \hat{\otimes} L^{1}(G)\right)^{*}$ (where the $l^{1}\left(\mathbb{F}_{0}\right)$-action on $\left(L^{1}(G)\right.$ $\left.\hat{\otimes} L^{1}(G)\right)^{*}$ is induced by $A$ ). By Lemma 3.1 now, there is a discontinuous derivation from $l^{1}\left(\mathbb{F}_{0}\right)$ to $\left(l^{1}\left(\mathbb{F}_{0}\right) \hat{\otimes} l^{1}\left(\mathbb{F}_{0}\right)\right)^{*}$.

It follows from the theorem that, if all $l^{1}(G)$-module homomorphisms from $L^{1}(G)$ are continuous for every $G$, then the continuity of derivations problem for group algebras may be reduced to the case when $G=\mathbb{F}_{0}$. In the case of weighted convolution algebras there is no similar reduction of the derivations problem to a particular weight function. However, a similar argument to that above yields the following result. 
4.2 THEOREM. Let $\omega$ be a weight function and suppose that there is a discontinuous derivation from $L^{1}(\omega)$ to a Banach $L^{1}(\omega)$-bimodule $X$. Then there is either a discontinuous $l^{1}(\omega)$-module homomorphism from $L^{1}(\omega)$ to $\left(L^{1}(\omega) \hat{\otimes} L^{1}(\omega)\right)^{*}$ or there is a discontinuous derivation from $l^{1}(\omega)$ to $\left(l^{1}(\omega) \hat{\otimes} l^{1}(\omega)\right)^{*}$.

We will conclude by showing that certain derivations from group algebras are continuous. Recall [2, Section 43] that an $L^{1}(G)$-bimodule $X$ is said to be commutative if $f \cdot x=x \cdot f,\left(f \in L^{1}(G), x \in X\right)$. Proposition 43.14 of [2] shows that if $G$ is amenable and $X$ is a commutative Banach $L^{1}(G)$-bimodule, then there is no non-zero continuous derivation from $L^{1}(G)$ to $X$.

4.3 THEOREM. Let $G$ be a locally compact group and $X$ be a commutative Banach $L^{1}(G)$-bimodule. Then every derivation from $L^{1}(G)$ to $X$ is continuous.

Proof. Put $Z=\left\{z \in\left(L^{1}(G) \hat{\otimes} L^{1}(G)\right)^{*} \mid f \cdot z=z \cdot f,\left(f \in L^{1}(G)\right)\right\}$. Then $Z$ is a $w^{*}$-closed subspace of $\left(L^{1}(G) \hat{\otimes} L^{1}(G)\right)^{*}$.

The argument used in Lemma 3.1 shows that, in order to prove the theorem, it will suffice to show that every derivation from $L^{1}(G)$ to $\left(L^{1}(G) \hat{\otimes} L^{1}(G)\right)^{*}$ whose range is contained in $Z$ is continuous. Let $D$ be such a derivation. Then by (3.4) there is a unique derivation $\bar{D}$ from $M(G)$ into $\left(L^{1}(G) \hat{\otimes} L^{1}(G)\right)^{*}$ which extends $D$. Because of the form of $\bar{D}$, and the fact that $Z$ is $w^{*}$-closed, the range $\bar{D}$ is contained in $Z$. (Note that $Z$ is not necessarily a submodule of $\left(L^{1}(G) \hat{\otimes} L^{1}(G)\right)^{*}$ and so, strictly speaking, $D$ and $\bar{D}$ are not derivations into $Z$.)

Now $\mathbf{S}(D)$ is a closed submodule of $\left(L^{1}(G) \hat{\otimes} L^{1}(G)\right)^{*}$ and is contained in $Z$. Hence, $\mathbf{S}(D)$ is a closed, commutative submodule of $\left(L^{1}(G) \hat{\otimes} L^{1}(G)\right)^{*}$. Put $I=\left\{f \in L^{1}(G) \mid f \cdot \mathbf{S}(D)=(0)\right\}$.

Let $x$ and $y$ be in $G$. Then for each $s$ in $\mathbf{S}(D),(x y)^{-} \cdot s=\bar{x} \cdot(\bar{y} \cdot s)=(\bar{y} \cdot s)$ $\cdot \bar{x}=(s \cdot \bar{y}) \cdot \bar{x}=s \cdot(y x)^{-}=(y x)^{-} \cdot s$. Hence, $\left(G^{\prime}\right)^{-}$, the closure of the commutator subgroup of $G$, acts trivially on $\mathbf{S}(D)$. It follows that if we define $J$ to be the closed, two-sided ideal in $L^{1}(G)$ generated by $\left\{f-\bar{x} * f \mid x \in\left(G^{\prime}\right)^{-}, f \in L^{1}(G)\right\}$, then $J \subseteq I$.

Suppose that $I$ has infinite codimension in $L^{1}(G)$. Then $I / J$ has infinite codimension in $L^{1}(G) / J$, which is isomorphic to the commutative group algebra $L^{1}\left(G /\left(G^{\prime}\right)^{-}\right)$, by [13, Section 1]. Hence by condition (i) at the beginning of Section 2, there are sequences $\left(a_{n}\right),\left(b_{n}\right)$ in $L^{1}(G)$ such that $b_{n+1} * a_{1} * \cdots * a_{n} \notin I$ but $b_{n+1} * a_{1} * \cdots * a_{n} * a_{n+1} \in I$. We may now deduce a contradiction to Lemma 1.4 just as in Theorem 2 of [11]. Therefore $I$ has finite codimension in $L^{1}(G)$.

Since $L^{1}(G) / I$ is commutative and finite dimensional, there are multiplicative linear functionals $\chi_{1}, \chi_{2}, \ldots, \chi_{n}$ on $L^{1}(G)$ such that $I=\bigcap_{j=1}^{n} \operatorname{ker}\left(\chi_{j}\right)$. For each 
$\chi_{j}$, define an element $\chi_{j} \otimes \chi_{j}$ in $\left(L^{1}(G) \otimes L^{1}(G)\right)^{*}$ by putting

$$
\left(\chi_{j} \otimes \chi_{j}\right)(f \otimes g)=\chi_{j}(f) \chi_{j}(g) \quad\left(f, g \in L^{1}(G)\right),
$$

and then extending $\chi_{j} \otimes \chi_{j}$ to the rest of $L^{1}(G) \hat{\otimes} L^{1}(G)$ by linearity and continuity. Then $\mathbf{C}\left(\chi_{j} \otimes \chi_{j}\right)$ is a one-dimensional submodule of $\left(L^{1}(G) \hat{\otimes}\right.$ $\left.L^{1}(G)\right)^{*}$ and $\mathbf{C}\left(\chi_{j} \otimes \chi_{j}\right)=\left\{z \in\left(L^{1}(G) \hat{\otimes} L^{1}(G)\right)^{*} \mid f \cdot z=z \cdot f=\chi_{j}(f) z,(f \in\right.$ $\left.\left.L^{1}(G)\right)\right\}$. Now, since $\mathbf{S}(D)$ is a commutative submodule of $\left(L^{1}(G) \hat{\otimes} L^{1}(G)\right)^{*}$ and $I$ is the annihilator of $\mathbf{S}(D)$, it follows that

$$
\mathbf{S}(D)=\bigoplus_{j=1}^{n} \mathbb{C}\left(\chi_{j} \otimes \chi_{j}\right) .
$$

In particular, $\mathbf{S}(D)$ is finite dimensional.

Put $P=\left\{\psi \in L^{1}(G) \hat{\otimes} L^{1}(G) \mid s(\psi)=0,(s \in \mathbf{S}(D))\right\}$. Then $P$ is a closed $L^{1}(G)$-submodule of $L^{1}(G) \hat{\otimes} L^{1}(G)$. For each $\psi$ in $P$ define a linear functional $T_{\psi}$ by $T_{\psi}(f)=D(f)(\psi),\left(f \in L^{1}(G)\right)$. Then $T_{\psi}$ is continuous because $T_{\psi}=$ $R_{\psi} \circ Q \circ D$, where $Q$ is the quotient map of $\left(L^{1}(G) \hat{\otimes} L^{1}(G)\right)^{*}$ by $\mathbf{S}(D)$, so that $Q \circ D$ is continuous, and $R_{\psi}$ is the continuous linear functional on $\left(L^{1}(G) \hat{\otimes}\right.$ $\left.L^{1}(G)\right)^{*} / \mathbf{S}(D)$ defined by $R_{\psi}(z+\mathbf{S}(D))=z(\psi)$. Hence, for each $f$ and $a$ in $L^{1}(G)$ and $\phi$ in $P$,

$$
\begin{aligned}
D(f * a)(\phi) & =T_{\phi}(f * a)=T_{\phi}\left(\int_{G} f(x) \bar{x} * a d x\right) \\
& =\int_{G} f(x) T_{\phi}(\bar{x} * a) d x \quad(\text { by }[4, \text { Theorem III 2.19(c)] }) \\
& =\int_{G} f(x) D(\bar{x} * a)(\phi) d x .
\end{aligned}
$$

Let $f$ be in $L^{1}(G)$ and $\psi$ be in $P$. Then, since $L^{1}(G)$ has a left bounded approximate identity for $L^{1}(G) \hat{\otimes} L^{1}(G)$ and $P$ is a closed $L^{1}(G)$-submodule, there are elements $a$ in $L^{1}(G)$ and $\phi$ in $P$ such that $\psi=a \cdot \phi$. Hence

$$
\begin{aligned}
D(f)(\psi) & =D(f)(a \cdot \phi) \\
& =(D(f) \cdot a)(\phi) \\
& =(-f \cdot D(a)+D(f * a)(\phi) \\
& =\int_{G} f(x)(-\bar{x} \cdot D(a)+D(\bar{x} * a))(\phi) d x \\
& =\int_{G} f(x)(\bar{D}(\bar{x}) \cdot a)(\phi) d x \\
& =\int_{G} f(x) \bar{D}(\bar{x})(\psi) d x .
\end{aligned}
$$


Now for each $x$ in $G,\langle x\rangle$, the subgroup of $G$ generated by $x$, is abelian and thus is amenable. Hence, by Proposition 43.14 of [2], the restriction of $\bar{D}$ to $L^{1}(\langle x\rangle)$ is zero. In particular, $\bar{D}(\bar{x})=0$ for every $x$ in $G$. Therefore, by (4.3), $D(f)(\psi)=0$ for every $f$ in $L^{1}(G)$ and every $\psi$ in $P$. That is, $D(f)(\psi)=0$ for every $\psi$ which is annihilated by $\mathbf{S}(D)$. Hence, since $\mathbf{S}(D)$ is finite dimensional, $D(f)$ is in $\mathbf{S}(D)$ for every $f$ in $L^{1}(G)$. Thus, by (4.1), $D$ is a direct sum of point derivations from $L^{1}(G)$ to the one-dimensional bimodules $\mathbf{C}\left(\chi_{\psi} \otimes \chi_{\psi}\right), j=$ $1,2, \ldots, n$. However, by the theorem in [18], there are no non-zero point derivations on $L^{\mathbf{l}}(G)$. Therefore $D$ is continuous.

Note added in proof. Since writing this paper I have solved problem (C) in Section 2. As anticipated in Section 2, the solution uses Lemma 2.1, and a result similar to the conjecture (E). A consequence of this is that Theorem 4.1 is strengthened because the first alternative in its conclusion cannot occur. The solution, together with some results which were originally intended for the reference [19], will now appear in two papers

[a] 'The continuity of derivations from group algebras: factorizable and connected groups',

[b] 'The continuity of left $l^{1}(G)$-module homomorphisms from $L^{1}(G)$ '.

\section{References}

[1] W. G. Bade and H. G. Dales, 'Norms and ideals in radical convolution algebras', J. Functional Analysis 41 (1981), 77-109.

[2] F. F. Bonsall and J. Duncan, Complete normed algebras (Springer-Verlag, New York, 1973).

[3] H. G. Dales, 'Automatic continuity: a survey', Bull. London Math. Soc. 10 (1978), 129-183.

[4] N. Dunford and J. T. Schwartz, Linear operators, part I (Interscience, New York, 1957).

[5] R. Engelking, General topology (Polish Scientific Publishers, Warsaw, 1977).

[6] G. I. Gaudry, 'Multipliers of weighted Lebesgue and measure spaces', Proc. London Math. Soc. (3) 19 (1969), 327-340.

[7] F. Ghahramani, 'Homomorphisms and derivations of weighted convolution algebras', $J$. London Math. Soc. (2) 21 (1980), 149-161.

[8] S. Grabiner, 'Weighted convolution algebras on the half line', J. Math. Anal. Appl. 83 (1981), 531-553.

[9] E. Hewitt and K. A. Ross, Abstract harmonic analysis, Vols. I and II (Springer-Verlag, New York, 1979).

[10] E. Hille and R. S. Phillips, Functional analysis and semigroups, (American Mathematical Society, Providence, R. I., 1957).

[11] N. P. Jewell, 'Continuity of module and higher derivations', Pacific J. Math. 68 (1977), 91-98.

[12] B. E. Johnson, 'Continuity of centralizers on Banach algebras', J. London Math. Soc. 41 (1966), 639-640.

[13] H. Reiter, $L^{1}$-algebras and Segal algebras (Lecture Notes in Math. 231, Springer-Verlag, Berlin, 1971)). 
[14] W. Rudin, Fourier analysis on groups (Interscience, New York, 1962).

[15] W. Rudin, Real and complex analysis (2nd edition, McGraw-Hill, New York, 1974).

[16] A. M. Sinclair, Automatic continuity of linear operators (Cambridge University Press, Cambridge, 1976).

[17] G. A. Willis, Ph.D. Thesis, University of Newcastle-upon-Tyne, 1981.

[18] G. A. Willis, 'Factorization in codimension one ideals of group algebras', Proc. Amer. Math. Soc. 86 (1982), 599-601.

[19] See Note added in proof.

Department of Mathematics, Statistics and Computing Science

Dalhousie University

Halifax

Nova Scotia B3H $4 \mathrm{H} 8$

Canada 